\title{
Aplicación de análisis de pastas macroscópicos, petrográficos y de composición de elementos químicos al problema de la procedencia de cerámica en el Período Alfarero Temprano de Chile central y Cuyo, Argentina
}

Lorena Sanhueza R., ${ }^{1}$ Fernanda Falabella G., ${ }^{2}$ Eugenia Fonseca P. ${ }^{3}$ Y Oscar Andonie Z. ${ }^{4}$

\section{RESUMEN}

Se presenta la aplicación de una secuencia analítica, que incluye análisis macroscópico de pastas bajo lupa binocular, análisis petrog ráficos y análisis por activación neutrónica instrumental para investigar la procedencia de cerámica del sitio El Indígeno (Argentina) y evaluar la posibilidad de que ésta llegue desde la región central de Chile. Los resultados sugieren que los materiales cerámicos de este sitio no sólo tienen una apariencia formal y decorativa que permite integrarlos dentro del Complejo Llolleo, sino que también comparten el mismo patrón tecnológico. No obstante lo anterior, la comparación de la composición de elementos con el sitio La Granja en la cuenca del río Cachapoal, uno de los probables sitios de procedencia, indica que no comparten las mismas fuentes de materias primas.

Palabras claves : cerámica - procedencia - análisis de pasta - petrog rafía - análisis por activación neutrónica.

\begin{abstract}
In this study, we use binocular, petrographic and instrumental neutron activation analyses to study ceramic pastes from El Indígeno (Argentina) in order to determine their provenance and to evaluate if they are reaching the site from central Chile. The results show that the ceramic context from El Indígeno not only shares morphological and decorative characteristics with Llolleo pottery from Chile, but also their technological pattern. The elemental composition differs from that of ceramics from La Granja, a Llolleo archaeological site on the Cachapoal river basin, which was thought as a potencial source for the El Indigeno ceramic material, indicating that they don't share the same clay sources.
\end{abstract}

Key words: pottery - provenience - pastes analyses petrography - neutron activation analyses.

Recibido: abril 2004. Manuscrito revisado aceptado: julio 2004.

1 Departamento de Antropología, Facultad de Ciencias Sociales, Universidad de Chile. Ignacio Carrera Pinto 1045, Ñuñoa, Santiago, CHILE. Email: loresan@uchile.cl

\section{Introducción}

La determinación de la procedencia de artefactos en arqueología se ha tratado desde distintos ángulos, entre los cuales el estilo de éstos ha sido uno de los más utilizados. Para la cerámica se ha supuesto que vasijas que comparten aspectos formales y decorativos podrían tener un origen común. Sin embargo, si bien una semejanza formal permite generar una propuesta en este sentido, no permite, por sí sola, discriminar si estamos frente a una copia o frente a un objeto trasladado desde su lugar de origen. Para discriminar entre estas dos opciones hay que recurrir al análisis de las materias primas con que fueron elaborados los artefactos.

En el caso de los materiales líticos (p.e., obsidiana) la materia prima no sufre grandes transformaciones con la producción del artefacto, por lo que el análisis de éste permite relacionarlo de manera bastante precisa con la fuente de materia prima respectiva. En el caso de la cerámica, el proceso de producción puede alterar las características de las fuentes originarias mediante procesos de limpieza, de mezcla de materias primas de distintas fuentes o la adición de desgrasantes. La relación entre el artefacto terminado y la fuente de materia prima es, entonces, menos directa y la solución del problema de la procedencia más complejo. En este escenario, creemos que la utilización de distintas metodologías pueden mejorar sustantivamente los resultados de nuestros estudios.

2 Departamento de Antropología, Facultad de Ciencias Sociales, Universidad de Chile. Ignacio Carrera Pinto 1045, Ñuñoa, Santiago, CHILE. Email: ffala@entelchile.net

3 Departamento de Laboratorio, SERNAGEOMIN. Tiltil 1993, Nuñoa, Santiago, CHILE. Email: efonseca@sernageomin.cl

4 Departamento Aplicaciones Nucleares, Comisión Chilena de Energía Nuclear. Nueva Bilbao 12501, La Reina, Santiago, CHILE. Email: oandonie@cchen.cl 
Existen diversos procedimientos para el análisis de las materias primas cerámicas, las que pueden ser más o menos elaboradas y más o menos costosas, y que apuntan a sus distintos componentes principales: la arcilla y los antiplásticos o desgrasantes.

Los análisis macroscópicos de pastas pueden realizarse en una muestra grande de fragmentos o también si se cuenta con el tiempo suficiente, sobre todo el material en estudio, ya que sólo se requiere de una lupa binocular y de una persona entrenada para realizar el análisis. La observación con lupa permite formarse una idea de ciertas características de la matriz, pero sobre todo de la naturaleza y características de los áridos presentes en ella. Una persona con experiencia incluso puede llegar a realizar una identificación mineralógica de gran parte de ellos, así como se puede estimar tamaño, densidad y grado de transporte.

Los análisis petrográficos, por su parte, requieren de un microscopio de luz polarizada, de la realización de cortes delgados de los fragmentos que se quieren analizar y de una persona especializada en la identificación de rocas y minerales. Este análisis es, por lo tanto, más costoso y lento que el anterior $\mathrm{y}$ es aplicado, por lo general, a una muestra mucho menor que se selecciona a partir del análisis anterior. La petrografía apunta también principalmente a los áridos que componen la cerámica.

El análisis por activación neutrónica (INAA) es una técnica mucho más sofisticada, que requiere de un reactor nuclear y que apunta a la determinación de la concentración de los elementos químicos en la materia prima, los que caracterizan tanto los áridos como la arcilla que compone la matriz. Es, por supuesto, una técnica mucho más costosa y que también es aplicada a una muestra pequeña de los materiales.

Creemos que estos procedimientos se complementan y potencian porque: a) apuntan a los distintos componentes de la cerámica, por lo que se obtiene información de naturaleza distinta, y b) las primeras constituyen antecedentes importantes para la realización de análisis más sofisticados y caros como los INAA, permitiendo que la muestra analizada sea representativa.

En este trabajo presentaremos los resultados de la aplicación de esta secuencia analítica para deter- minar la procedencia de cerámica supuestamente foránea del sitio El Indígeno, ubicado en la vertiente oriental de los Andes.

\section{Antecedentes del problema arqueológico}

El sitio El Indígeno se encuentra a 3500 m.snm, en un valle interandino, entre las cabeceras de los ríos Diamante y Atuel, al oriente de la línea divisoria de aguas de la cordillera de los Andes, muy próximo al Paso Las Leñas. Se trata de un sitio muy especial que, por las condiciones de altura, sólo puede ser ocupado durante pocos meses en verano. Concentra unos 140 recintos pircados y, si bien existen otros sitios similares en esa zona, es el que tiene mayor abundancia de materiales y estructuras (Lagiglia 1997).

Lagiglia (1997) ha utilizado un modelo etnohistórico para explicar las ocupaciones de este sitio. Su hipótesis es que éste estaría formando parte del circuito de movilidad de grupos cazadoresrecolectores cordilleranos que se mueven entre el piedemonte y llanuras bajas orientales (1600 m.snm), de ocupación invernal, y los valles interandinos entre los 2000 y $4000 \mathrm{~m}$ de altura en el verano. Estos mismos cazadores-recolectores bajarían ocasionalmente hacia la vertiente occidental para intercambiar productos con grupos de los valles (Maipo y/o Cachapoal/Tinguiririca).

Parte importante del sustento de esta hipótesis radica en los materiales cerámicos del sitio. La cerámica más abundante corresponde a fragmentos de vasijas de un tipo que ha sido denominado "Overo" que es considerado por Lagiglia (1997) como el tipo tradicional de la cordillera. Se trata fundamentalmente de fragmentos de ollas alisadas con asa ("Overo ordinario"), algunas de las cuales tienen incisiones en el cuello ("Overo inciso"). Este tipo es similar a una cerámica que aparece en los sitios hacia el piedemonte oriental, bajo los 2000 m.snm, que se ha denominado "Nihuil", diferenciándose fundamentalmente por el tono de la pasta y textura (menos rojiza y menos deleznable que la del Overo). En El Indígeno, junto a esta cerámica Overo, pero en muy baja frecuencia, se reconocieron fragmentos que fueron considerados "foráneos" por sus características formales. Un grupo de éstos fueron postulados como pertenecientes a la cerámica de grupos "Llolleo" del Período Alfarero Temprano (PAT) de Chile (200-1000 DC). 
Desde el punto de vista del componente cerámico del sitio, en este modelo se plantea una hipótesis de procedencia bastante clara. Las vasijas Overo serían bienes propios de estos cazadoresrecolectores y su producción se realizaría en algún lugar del circuito de movilidad, ya sea en el valle interandino donde se localiza El Indígeno durante su ocupación estival y/o en los faldeos o valles de la vertiente oriental de los Andes. Las vasijas "foráneas" serían llevadas al sitio por los mismos cazadores recolectores como producto de sus intercambios con los grupos occidentales y, por lo tanto, serían producidas en algún lugar hacia el poniente de la cordillera de los Andes.

Una revisión de la cerámica del sitio realizada recientemente en el marco de una investigación conjunta entre investigadores de Chile y Argentina mostró que el contexto Llolleo en El Indígeno parece ser mucho más importante y abundante que lo originalmente estimado. Esta revisión incluyó un análisis detallado de las formas y decoraciones presentes en el sitio, de manera de establecer de manera cierta las similitudes de algunos materiales con los del Complejo Llolleo (Figura 1).

De esta manera se registraron diversos componentes del contexto cerámico Llolleo como: a) fragmentos negro y café pulido delgado; b) bordes reforzados; c) cuellos inciso reticulado y asas mamelonares incisas, característicos de las ollas Llolleo inciso reticulado; d) ciertos motivos y configuraciones incisas (que llamamos "inciso complejo") que son similares a fragmentos que aparecen en los sitios Llolleo más meridionales de la cuenca del Cachapoal-Tinguiririca; e) fragmentos con incisión anular en la base del cuello; f) fragmentos pulidos pintados rojo; g) incisos y pintados rojo (Tabla 1).

Junto a estos elementos cerámicos se registraron cuentas de collar y pipas de greda, también características del Complejo Llolleo.

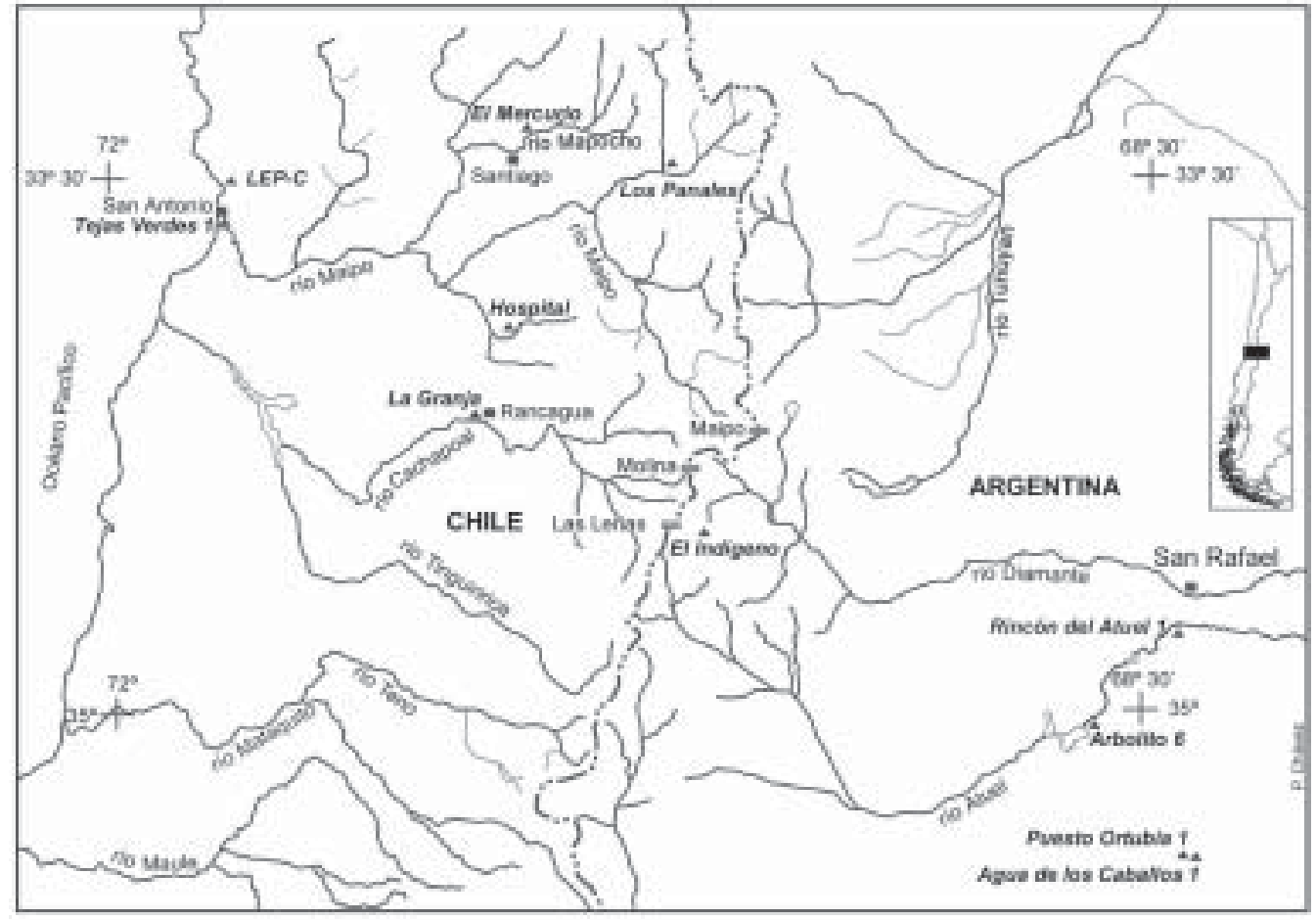

Paso condilerano -

Sitio arqueologice .

Limite internacional

Figura 1. Mapa que muestra la ubicación de los sitios arqueológicos mencionados en el texto. 


\begin{tabular}{|lcc|}
\hline Material & N & \%* \\
\hline Formas & & \\
fragmentos negro y café pulido & 93 & 2.1 \\
bordes reforzados & 28 & 0.6 \\
& & \\
Decoraciones & & \\
inciso reticulado & 57 & 1.3 \\
inciso lineal & 29 & 0.7 \\
inciso complejo & 33 & 0.7 \\
incisión anular en base de cuello & 4 & 0.09 \\
inciso Overo & 5 & 0.1 \\
mamelón inciso sobre asa cinta & 10 & 0.2 \\
mamelón inciso en cuerpo & 7 & 0.2 \\
rojo pintado pulido & 112 & 2.5 \\
rojo + inciso & 3 & 0.07 \\
\hline
\end{tabular}

Tabla 1. Fragmentos con características de forma, superficie y/o decoración Llolleo en el sitio El Indígeno. *\% de referencia calculado en función de un total de 4402 fragmentos analizados, que no corresponde al total del material recuperado del sitio.

Algunos de estos componentes, que reconocemos como parte del estilo alfarero Llolleo, habían sido considerados por Lagiglia (1997) dentro del tipo Overo como los fragmentos de vasijas incisoreticuladas, los bordes reforzados y los incisos complejos. Si a esto agregamos que los fragmentos Overo sin decoración, por sus formas y tratamientos de superficie, son indiferenciables de fragmentos Llolleo no decorados, resulta que el componente alfarero Llolleo puede tener mayor relevancia en el sitio El Indígeno que lo previamente estimado.

La información de los atributos formales y decorativos llevó a identificar parte importante del contexto alfarero de El Indígeno con el estilo alfarero Llolleo. Dado que pensamos que es poco probable que se haya producido cerámica durante las ocupaciones estivales en este sitio de alta cordillera $^{5}$, apoyados en la evidencia estilística hemos propuesto un modelo alternativo, en el cual El Indígeno podría ser parte de un circuito de movilidad de los grupos Llolleo de Chile central. Esto implicaría, en la perspectiva de la cerámica, no sólo que parte importante del componente Overo del sitio El Indígeno podría ser Llolleo (además de lo que tradicionalmente ha sido con-

5 Los estudios etnográficos en comunidades alfareras tradicionales han develado características, en la organización y procesos de manufactura, que hacen pensar que es poco probable desarrollar esta actividad en ocupaciones temporales con escasez de combustible (Arnold 1985). siderado como foráneo), sino que tendría una procedencia occidental, desde la cuenca y precordillera de Santiago, accediendo por el valle del Maipo o desde la de Rancagua, subiendo por el río Cachapoal, Las Leñas u otro.

\section{Materiales y método}

De acuerdo a nuestra propuesta, la mayor parte de la cerámica de El Indígeno debería presentar similitudes a nivel de materias primas con la cerámica Llolleo. Para poder comprobar esta similitud era necesario comparar la cerámica del primero con la que se encuentra en los sitios Llolleo de los valles de Chile. Por otro lado, también consideramos necesario realizar una comparación similar con contextos del piedemonte oriental de Arg entina, para descartar su similitud con contextos de El Indígeno.

En el caso de Chile existían estudios de varios sitios Llolleo de costa (p.e., LEP-C y Tejas Verdes 1), valle (p.e., El Mercurio, La Granja y Hospital) y cordillera (p.e., Los Panales), que sirvieron de referente de comparación (Falabella y Planella 1979 y 1987; Planella et al. 1996 Ms; Sanhueza 1997; Falabella 2000; Sanhueza et al. 2003). Se utilizaron en forma especial los datos de pasta y petrografía de los sitios El Mercurio y La Granja de las cuencas de Santiago y Rancagua, respectivamente.

En el caso de Argentina, la zona de estudio lamentablemente no dispone de muchos antecedentes arqueológicos. Hay reconocimiento sólo de algunos sitios que podrían pertenecer al Período Alfarero Temprano, algunos con recolecciones de superficie pero escasas excavaciones y controles cronológicos. Para este estudio se analizó la cerámica del sitio Arbolito-6, como un referente de un contexto cerámico de las zonas bajas dentro del probable circuito de movilidad de los habitantes de El Indígeno y de las pastas de esa región. Lamentablemente, el otro sitio excavado del Período Alfarero Temprano, Rincón del Atuel, tenía escasa cerámica (146 fragmentos) y no sirvió para el estudio. Los otros sitios analizados, Puesto Ortubia-1 y Agua de los Caballos-1, resultaron muy tardíos (1500 DC). Para estos contextos también se realizó análisis de formas y decoraciones, de manera de contar con la misma información para todos los contextos estudiados (sitio El Indígeno y sitios de los valles chilenos). 
La metodología contempló análisis de pastas con lupa binocular, de petrografía y de activación neutrónica.

\section{Los análisis macroscópicos con lupa binocular}

Para estos análisis se siguió un procedimiento estándar, que incluyó la realización de un corte fresco en cada fragmento examinado e inspección bajo lupa binocular con 10 aumentos. Se clasificó el universo de estudio en familias de pasta, que agrupan a patrones de pastas de acuerdo al origen geológico de sus áridos (volcánicos, graníticos). Se utilizaron los patrones de pastas definidos para sitios Llolleo de Chile para comparar con los patrones de pastas definidos en los sitios de la vertiente oriental.

En todos los sitios trabajamos con el criterio de revisar $30 \%$ de la cerámica. Esta es la muestra que tienen los sitios Llolleo de Chile que han sido estudiados (La Granja, El Mercurio, Hospital-6 y Los Panales).

Como antecedente es importante destacar que la cerámica Llolleo de Chile central tiene un claro patrón tecnológico para la preparación de la pasta que se reconoce bajo la lupa binocular y que se caracteriza por: a) una preferencia por áridos volcánicos básicos de caja de río (aunque existen otros materiales en la zona); b) una granulometría unimodal; c) densidad media-alta. Los grupos Llolleo optan por una alternativa entre otras posibles, y es así como a nivel de pastas se distinguen bien de otros grupos contemporáneos que habitan los mismos lugares y optan por otras fuentes de materias primas. En este sentido se trata de una tradición tecnológica fuertemente arraigada.

Los resultados de los análisis en El Indígeno indican que el patrón tecnológico Llolleo es el que domina en las muestras del sitio (Tabla 2). A este patrón pertenecen los fragmentos de atributos formales Llolleo, así como el grueso de la fragmentería clasificada como Overo alisada o pulida sin decoración. Por lo tanto, parecen corresponder, desde el punto de vista de las pastas, a un mismo conjunto. Es importante recalcar también que, si bien hay otros patrones de pasta en el sitio, éstos son minoritarios. En otras palabras, la mayor parte de la cerámica "Overo ordinario", que ha sido considerada como local, tiene las mismas pastas que la "foránea" y estas pastas son iguales (bajo lupa) a las de la tradición tecnológica Llolleo.

En el caso de Arbolito-6, existe una variabilidad de pastas mucho mayor que se reparten en términos de porcentajes (ningún grupo sobrepasa 20\%) y donde el patrón tecnológico Llolleo es sólo una opción más entre otras (Tabla 3). En este conjunto se distinguen dos grupos principales. La cerámica tipo Nihuil (considerada como la variante de valles bajos del Overo) posee pastas de patrón Llolleo (V2), lo que afirma la similitud reconocida por Lagiglia entre ésta y la cerámica Overo. La cerámica tipo Arbolito se caracteriza por presentar pastas de matrices claras (Vn-Vnc, X, BV) con antiplásticos de obsidiana y otros translúcidos.

La presencia de patrones de pasta similares a los Llolleo en la vertiente oriental de los Andes tiene varias explicaciones posibles, como la circulación de vasijas desde el occidente, las características de las fuentes de materias primas y la posibilidad de que distintos grupos compartan una tradición de manufactura.

Para avanzar en la solución de estas interrogantes se hizo imperativo recurrir a otras técnicas de análisis que nos permitiesen identificar los áridos y sus características mineralógicas (cristalización u otros), para poder relacionar la composición de la pasta con formaciones geológicas particulares y acceder a información sobre las arcillas.

\begin{tabular}{|lcccr|}
\hline Categoría & $\begin{array}{c}\text { Pasta familia V } \\
\text { (patrón Llolleo) }\end{array}$ & Pasta familia VB & Otras pastas & Total analizado \\
\hline fragmentos sin decoración & $591(69 \%)$ & $248(28.9 \%)$ & $18(2.1 \%)$ & 857 \\
fragmentos pulidos delgados & $36(57.1 \%)$ & $17(27 \%)$ & $10(15.9 \%)$ & 63 \\
bordes reforzados & $11(44 \%)$ & $10(40 \%)$ & $4(16 \%)$ & 25 \\
inciso reticulado & $34(68 \%)$ & $7(14 \%)$ & $9(18 \%)$ & 50 \\
inciso complejo & $24(96 \%)$ & & $1(4 \%)$ & 25 \\
\hline
\end{tabular}

Tabla 2. Familias de pasta del sitio El Indígeno. 


\begin{tabular}{|l|ccc|}
\hline Tipo cerámico & Patrón & N & \% \\
\hline \multirow{2}{*}{ Patrones del tipo Nihuil } & V1 & 46 & 9.8 \\
& V2 & $\mathbf{9 0}$ & $\mathbf{1 9 . 1}$ \\
& VBc & 54 & 11.4 \\
& & & \\
Patrones del tipo Arbolito & Vn & 88 & 18.6 \\
& Vnc & 29 & 6.1 \\
& BV & 44 & 9.3 \\
& X & 32 & 6.8 \\
& & & \\
Otros & Vc & 11 & 2.3 \\
& Vad & 20 & 4.2 \\
& VB & 20 & 4.2 \\
& Vt & 4 & 0.9 \\
& Bg & 2 & 0.4 \\
Total analizado & M & 3 & 0.6 \\
& otro & 29 & 6.1 \\
& & & \\
& & $\mathbf{4 7 2}$ & $\mathbf{1 0 0 . 0}$ \\
\hline
\end{tabular}

Tabla 3. Familias de pasta del sitio Arbolito-6.

\section{Los análisis de petrografía}

La metodología siguió los procedimientos estandarizados en el Laboratorio de SERNAGEOMIN para este tipo de análisis en cerámica arqueológica con cortes delgados y estudio cualitativo y semicuantitativo bajo microscopio de luz polarizada. El énfasis estuvo puesto en la identificación de elementos que se pudieran relacionar con determinadas formaciones geológicas.

Para el análisis petrográfico se escogió un ejemplar de cada uno de los grupos de pasta más frecuentes en los sitios. En este sentido trabajamos con el supuesto de que la observación de lupa binocular permite clasificar adecuadamente la cerámica en clases de pastas (patrones) donde los fragmentos incluidos en una clase comparten las características de los áridos y forman un conjunto relativamente homogéneo. De esta manera, un ejemplar analizado por petrog rafía sirve para caracterizar a su grupo.

La cantidad de muestras por sitio fue variable, dependiendo de las características del conjunto de cada sitio, y estuvo limitada por los costos. Se analizaron cinco muestras de La Granja, ocho de El Mercurio, 10 de El Indígeno y ocho de Arbolito- 6 , lo que constituye una cantidad pequeña de muestras por sitio.

En todos los sitios los resultados muestran la utilización dominante de áridos volcánicos que pue- den tener su origen en las formaciones geológicas de la cordillera de los Andes. Lamentablemente, las formaciones geológicas del área que estamos estudiando presentan características que no facilitan el trabajo de procedencia. Las formaciones de la cordillera de los Andes son bastante similares en el segmento norte-sur que estudiamos. Están dominadas por un volcanismo intermedio a básico, intercalado por intrusivos ácidos. Hacia el lado oriental hay mayor dominio de formaciones sedimentarias marinas (Vivallo et al. 1999). Sin embargo, el que haya una mayor presencia de un sustrato $u$ otro en una de las vertientes no es muy trascendente ya que los cursos fluviales que nacen en las altas cumbres intersectan el subsuelo y arrastran materiales, generando mezclas que pueden llegar a ser bastante similares en ambos lados de la cordillera.

Así, más que el tipo de mineral o roca presente en las muestras, nos sirvieron algunas de sus particularidades para establecer semejanzas y diferencias (Figuras 2 a 9). De esta manera pudimos reconocer que los volcánicos básicos de El Indígeno tenían ciertas características que eran las propias del sitio La Granja del valle del Cachapoal y que eran diferentes a como éstos se presentaban en los cortes de la cerámica del sitio El Mercurio del valle del Mapocho. Esto era observable en la propiedad de los basaltos con masa fundamental microlítica muy fina, en la característica afieltrada de los volcánicos ácidos por recristalización, en la presencia de piroclastos de la misma naturaleza, de epidotas de fuentes de alteración hidrotermal y en la presencia de oncolitos de origen sedimentario marino. De la misma manera la turmalina de brechas asociada a pórfidos cupríferos sobre los $4000 \mathrm{~m}$ está presente en La Granja, en todos los patrones V (o Llolleo) de El Indígeno y ausente en los otros patrones (VB) de ese sitio.

Respecto a los valles bajos del oriente, en Arbolito-6 se encontró turmalina sólo en un corte, el V2 de cerámica Nihuil (patrón tecnológico Llolleo), el que además presenta volcánicos con escaso transporte (por lo que serían fuentes de procedencia más cercanas a la cordillera). En este mismo sitio hay otras pastas volcánicas (V1) con áridos que evidencian mayor transporte (arrastradas por los ríos) y que por tanto podrían corresponder a fuentes más cercanas al sitio. La misma procedencia local denota la cerámica que tiene vidrio 


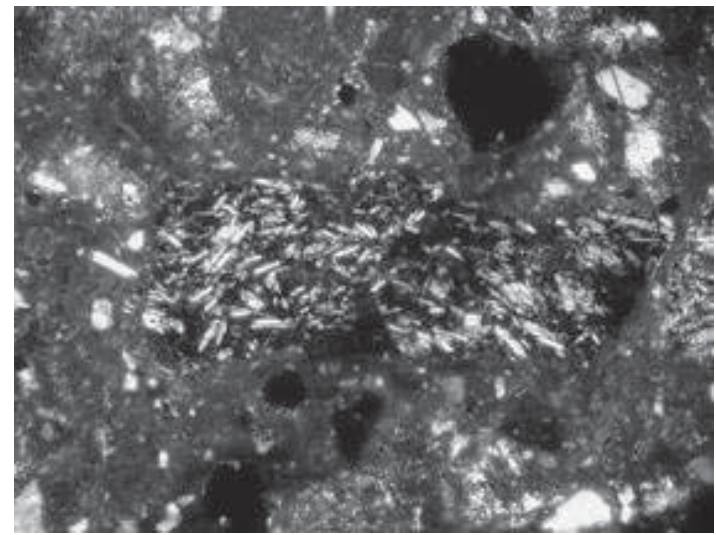

Figura 2. La Granja: Basaltos con masa fundamental microlítica muy fina.

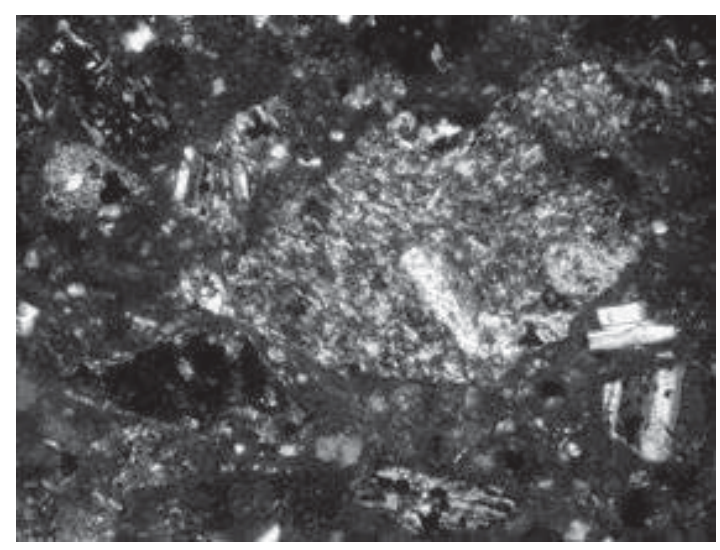

Figura 4. La Granja: Volcánicos ácidos afieltrados.



Figura 6. La Granja: Piroclastos.

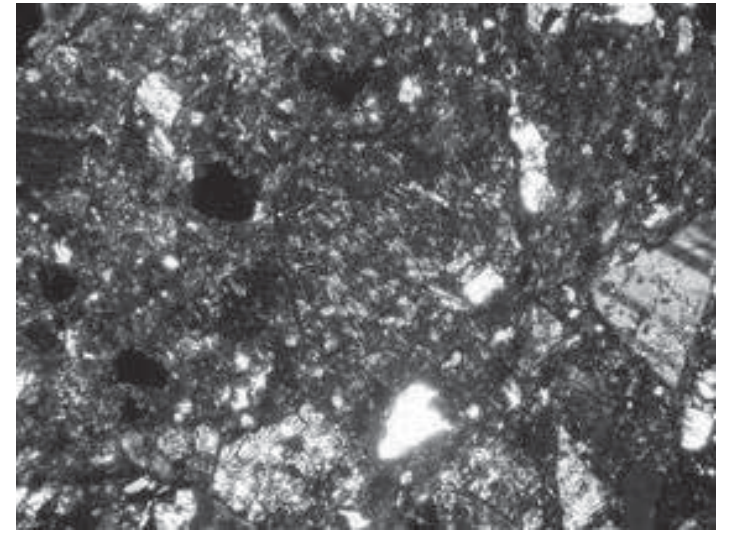

Figura 3. El Indígeno: Basaltos con masa fundamental microlítica muy fina.

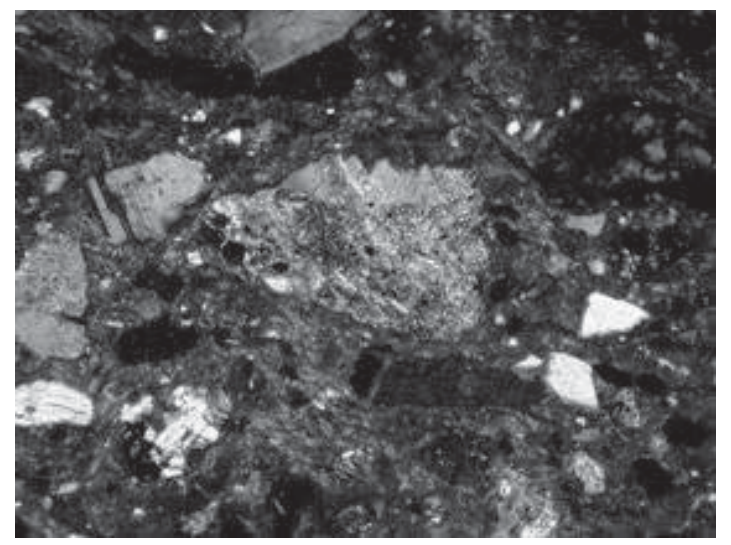

Figura 5. El Indígeno: Volcánicos ácidos afieltrados.

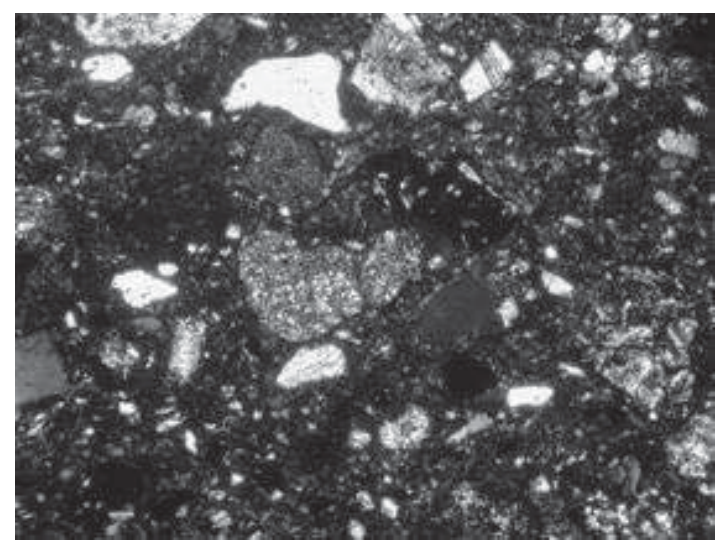

Figura 7. El Indígeno: Piroclastos. 


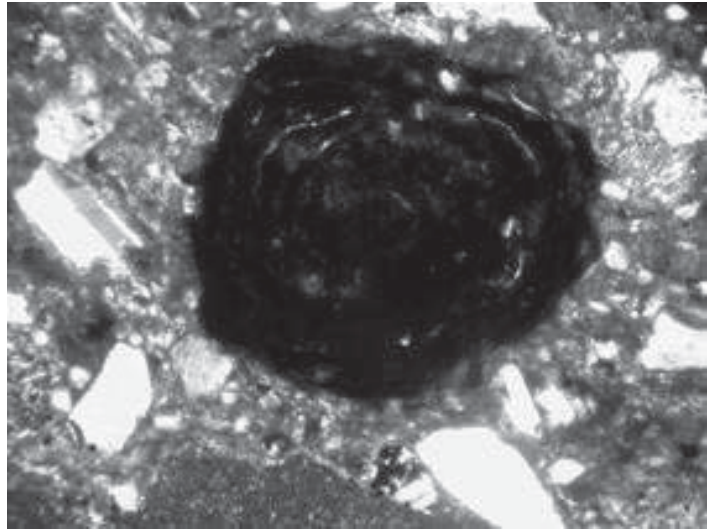

Figura 8. La Granja: Oncolito de origen sedimentario.

volcánico (obsidiana), ya que existe una formación con obsidiana próxima al sitio (El Peceño, V. Durán com. pers. 2003). Nada de esto se encuentra en El Indígeno ni en los sitios de Chile.

Desde el punto de vista metodológico, pese a la escasa diferenciación geológica regional, los análisis petrográficos entregaron un aporte significativo a través del detalle de las características de los volcánicos básicos y de elementos puntuales como los oncolitos, las obsidianas y el transporte. Estos datos corroboraron la naturaleza afín a fuentes cordilleranas de las materias primas, las que pueden estar localizadas en yacimientos primarios de zonas intercordilleranas o en yacimientos secundarios en terrazas de los cursos fluviales que bajan de los Andes.

En el caso de la cerámica de El Indígeno, si descartamos una manufactura local en el sitio mismo por la naturaleza temporal y los costos que implican los combustibles en un yacimiento de altura, con estos datos $-\mathrm{y}$ aplicando el criterio de abundancia- debemos pensar preferentemente en un aporte de vasijas desde el faldeo occidental, donde este tipo de pastas es muy abundante, más que del oriente, donde éstas son escasas. Además, sería más probable que vengan de la cuenca del Cachapoal (por la afinidad en las características de los áridos) que de la del Mapocho, con las que difieren.

\section{Análisis por activación neutrónica instrumental}

Con el análisis por activación neutrónica, que como ya dijimos, a diferencia de los anteriores

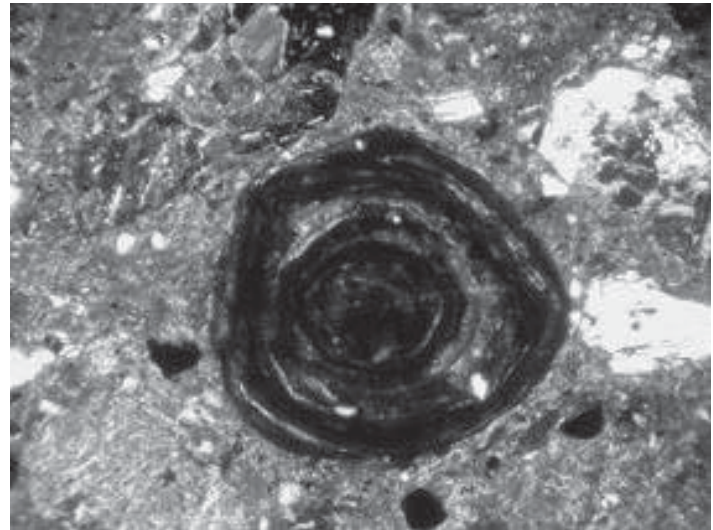

Figura 9. El Indígeno: Oncolito de origen sedimentario.

permite además acceder a información contenida en las arcillas, queríamos ver si la cerámica de El Indígeno (clasificada como Overo y con patrón tecnológico Llolleo dominante) se agrupaba con muestras de cerámica del sitio La Granja o formaba un conjunto discreto y distinto. Además, nos interesaba evaluar cómo se comportaban los fragmentos decorados dentro de estos conjuntos, ya que comparten algunas configuraciones que son bastante escasas a ambos lados de la cordillera. Esperábamos reconocer nubes discretas (grupos de referencia) si las fuentes de materias primas estaban localizadas y alejadas unas de otras, aunque compartieran características mineralógicas y geológicas.

Ya que no teníamos estudios previos de INAA de los contextos Llolleo de Chile ni de los sitios de Argentina, consideramos esta parte del trabajo como una experiencia exploratoria, que tuvo por objeto hacer una primera evaluación del comportamiento de las muestras (Tabla 4).

Se siguieron los procedimientos estandarizados en el laboratorio de la Comisión Chilena de Energía Nuclear, tanto para la obtención de la muestra como para la medición de la concentración de elementos químicos (Falabella y Andonie 2003).

Se exploró la existencia de agrupamientos con gráficos bivariados, se estimó si las diferencias en los distintos elementos eran estadísticamente significativas con test-t y se aplicaron análisis multivariados de Componentes Principales (PCA). En todos estos procedimientos se utilizaron los 17 elementos químicos con buena resolución: As, 


\begin{tabular}{|lcccccc|}
\hline Sitio/Tipo & Sin decoración & Bordes reforzados & Inciso reticulado & Inciso complejo & Inciso tipo Overo & Total \\
\hline La Granja & 7 & 2 & 3 & 4 & 1 & 14 \\
El Indígeno & 8 & $\mathbf{2}$ & $\mathbf{6}$ & $\mathbf{6}$ & $\mathbf{1}$ & $\mathbf{3 0}$ \\
Total & $\mathbf{1 5}$ & $\mathbf{2}$ & & & 16 \\
\hline
\end{tabular}

Tabla 4. Muestras cerámicas analizadas por activación neutrónica.

$\mathrm{Ce}, \mathrm{Co}, \mathrm{Cr}, \mathrm{Cs}, \mathrm{Eu}, \mathrm{Fe}, \mathrm{Hf}, \mathrm{La}, \mathrm{Lu}, \mathrm{Na}, \mathrm{Rb}, \mathrm{Sb}$, $\mathrm{Sc}, \mathrm{Sm}, \mathrm{Th}, \mathrm{Yb}$.

A nivel de comparación entre sitios, las muestras de El Indígeno y de La Granja no se agrupan (Tabla 5; Gráfico 1). El elemento que los diferencia mayormente es el arsénico (As), que segrega absolutamente las muestras por sitio. El arsénico está correlacionado con el rubidio y antimonio $(\mathrm{Rb}$ y $\mathrm{Sb})$, los que también presentan una diferencia estadísticamente significativa entre sitios (estos tres elementos, junto al Cs, son los que pesan en el Factor 2 del PCA). Estos resultados nos llevan a considerar las muestras de estos sitios como de fuentes distintas, a excepción de algunos decorados que se comentan más adelante.

Las muestras de El Indígeno forman una nube que incluye a los decorados, por lo que se podrían interpretar como de una fuente común (los que se alejan de esta nube son dos casos Overo sin decoración (\#4-9). A nivel intrasitio, las muestras de El Indígeno no presentan diferencias significativas en ninguno de los elementos, ni por tipo de cerámica.

Las muestras de La Granja tienen mayor variabilidad interna. Esto resulta coherente con la interpretación que se ha dado a este sitio en el sentido que correspondería a un lugar ritual de "juntas" al que accedería población de diferentes localidades de la región (Falabella at al. 2001). Pero, a diferencia de lo que ocurre en El Indígeno, a nivel intrasitio hay una diferencia importante: los incisos complejos y un inciso reticulado se separan del conjunto y se acercan a la nube de El Indígeno (Gráfico 2).

Si consideramos sólo las muestras de La Granja, vemos que estas diferencias se producen también en los elementos traza Ce, Eu, La y Sm, que denotan el componente arcilloso de la muestra (reflejados en el Factor 1 de los Gráficos 1 y 2). Los

\begin{tabular}{|lcc|}
\hline & \multicolumn{2}{c|}{$\begin{array}{c}\text { Rotated loading matrix } \\
\text { (varimax) }\end{array}$} \\
\cline { 2 - 3 } & PC 1 & PC 2 \\
\hline AS & 0.04 & 0.92 \\
CE & 0.91 & -0.06 \\
CO & 0.14 & 0.23 \\
CR & -0.08 & 0.09 \\
CS & -0.23 & 0.89 \\
EU & 0.42 & -0.14 \\
FE & 0.10 & -0.28 \\
HF & 0.85 & -0.09 \\
LA & 0.93 & 0.04 \\
LU & 0.13 & -0.02 \\
NA & 0.16 & -0.08 \\
RB & 0.12 & 0.75 \\
SB & 0.00 & 0.77 \\
SC & -0.55 & 0.02 \\
SM & 0.78 & 0.22 \\
TH & 0.90 & -0.06 \\
YB & 0.08 & 0.26 \\
Porcentaje de la varianza total & $26.32 \%$ & $18.09 \%$ \\
\hline
\end{tabular}

Tabla 5. Matriz de factores del análisis de componentes principales correspondiente a los Gráficos 1 y 2 .

cuatro incisos complejos no se agrupan entre sí, dando la impresión de marcar procedencias diversas. Por cierto esta dispersión de los incisos complejos incide en el menor agrupamiento que comentábamos de las muestras de La Granja.

Es demasiado prematuro comprometerse con una interpretación sobre las fuentes de procedencia en base a estos perfiles químicos de sólo dos sitios y con 30 fragmentos cerámicos. Si bien los resultados señalan que las muestras de El Indígeno tienen una procedencia diferente a las de La Granja y que los inciso complejos de Chile se comportan de una manera especial, no se puede descartar que la cerámica de El Indígeno pueda asimilarse a los perfiles de otros sitios Llolleo de Chile central, para los cuales aún no se han realizado análisis de activación neutrónica. 


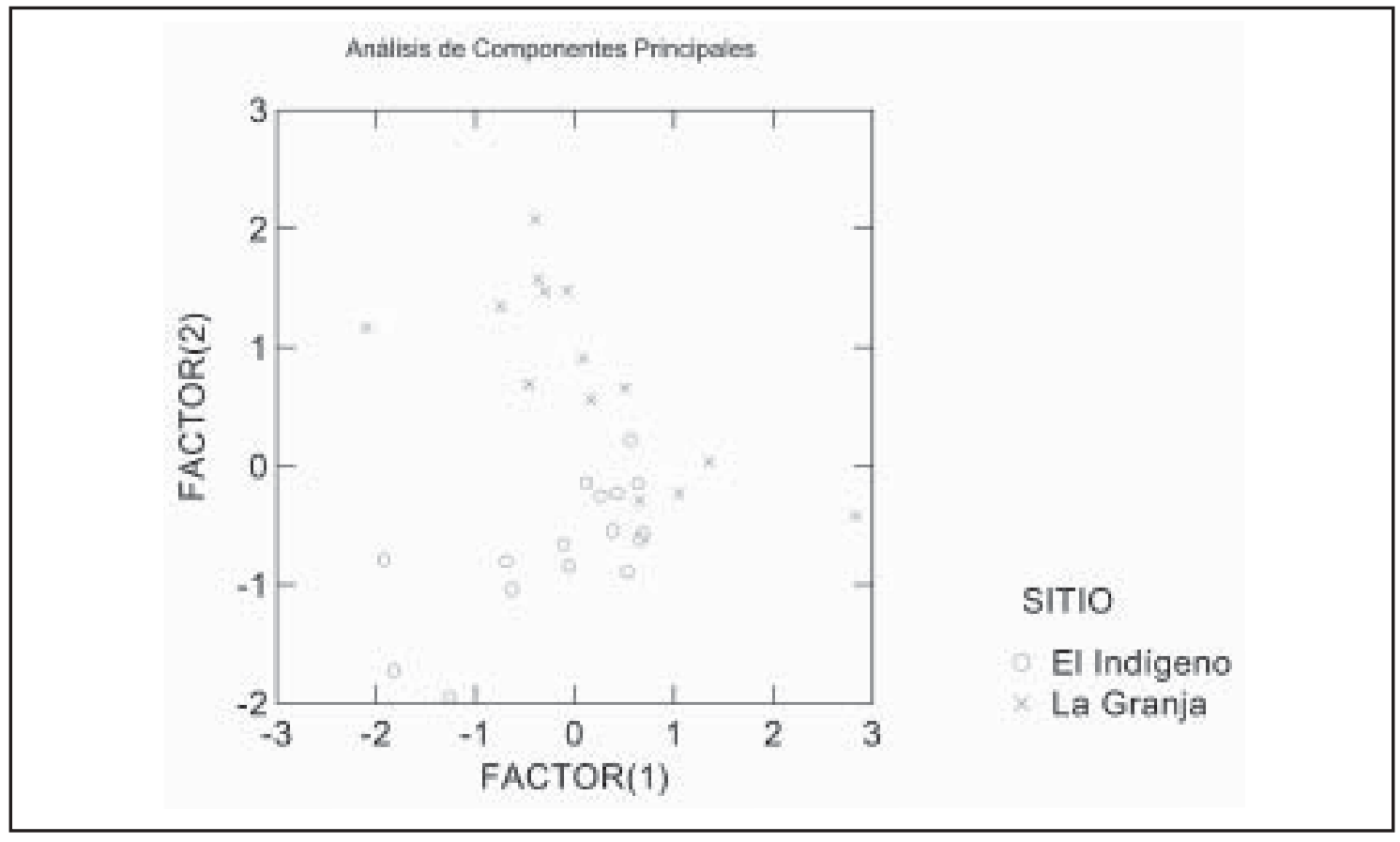

Gráfico 1. Disposición en el espacio bivariado de los factores 1 y 2 del análisis de PCA de los sitios El Indígeno y La Granja, codificado según la procedencia de la muestra.

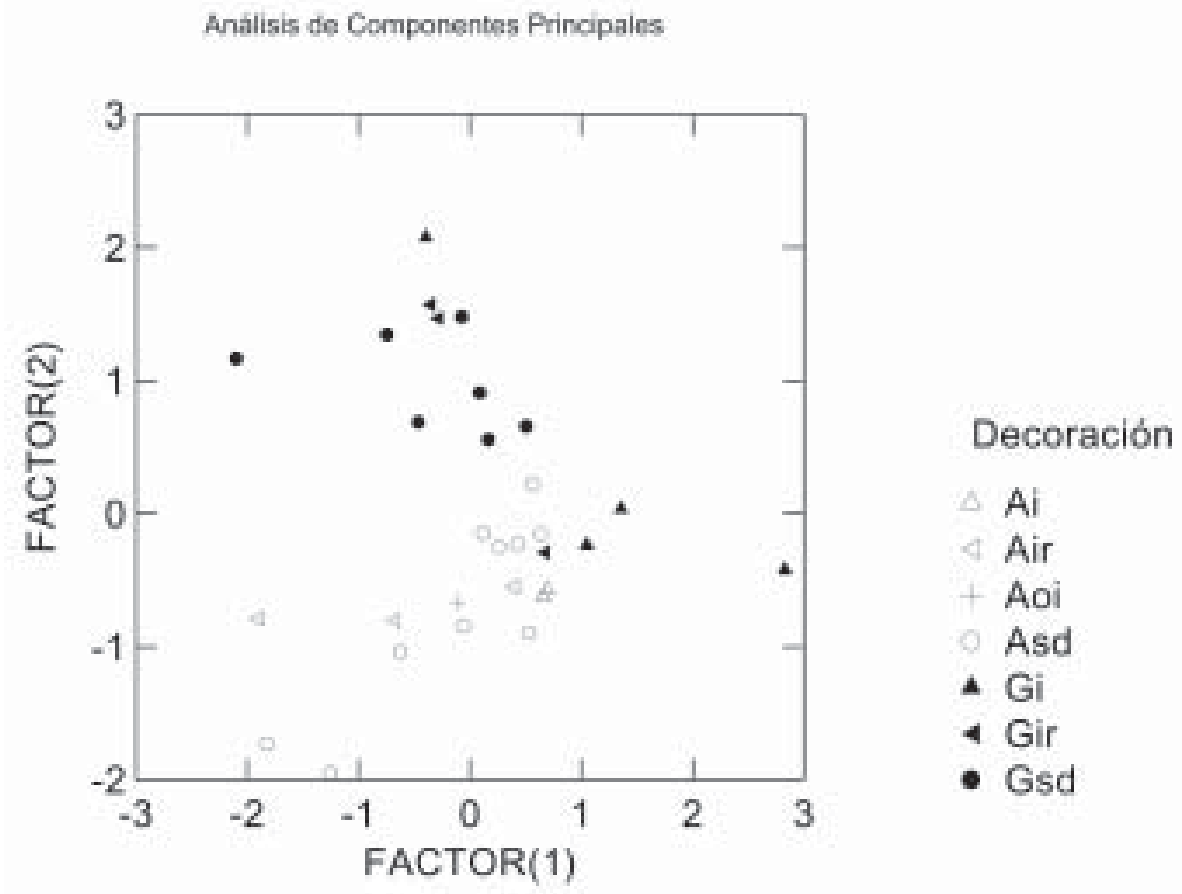

Gráfico 2. Disposición en el espacio bivariado de los factores 1 y 2 del análisis de PCA de los sitios El Indígeno y La Granja, codificado según la decoración. Ai=inciso complejo El Indígeno; Air=inciso reticulado El Indígeno; Aoi=inciso tipo Overo de El Indíg eno; Asd=El Indígeno sin decoración; Gi=inciso complejo La Granja; Gir=inciso reticulado La Granja; Gsd=La Granja sin decoración. 


\section{Conclusiones}

Si bien no logramos verificar nuestra hipótesis -que las vasijas de El Indígeno tienen una procedencia occidental- tampoco la hemos rechazado con estos análisis, por lo que aún quedan preguntas abiertas. Desde la perspectiva de los patrones tecnológicos de pasta, las evidencias apuntan más a una procedencia occidental. Desde la petrografía, se observan características comunes en el eje esteoeste a la altura del Diamante/Atuel-Cachapoal. La activación neutrónica segrega los conjuntos de El Indígeno y La Granja, pero al haberse analizado sólo un sitio de los valles chilenos, no puede descartarse completamente la posibilidad de que exista una relación a nivel de materias primas con algún otro sitio. Esto es coherente con la hipótesis que se maneja acerca de la producción cerámica al interior de Llolleo, que sería "a nivel de hogar", es decir, que cada unidad doméstica produce su conjunto de vasijas de manera independiente, aunque siguiendo un mismo estilo tecnológico (Sanhueza et al. 2003).

Creemos, a partir de esto, que nuestra hipótesis tiene argumentos para constituirse en una alternativa válida frente a la de Lagiglia (1997).

Juntar toda la información derivada de las pastas cerámicas en una síntesis en que todo "calce" y sea "coherente" no fue posible, pero es importante aclarar que esto resulta altamente improbable. La cerámica es heterogénea por definición. A diferencia de un material como la obsidiana (que deriva sólo de rocas ígneas, sufre fundamentalmente erosiones mecánicas que no alteran su mineralogía ni su composición química, está presente en lugares muy puntuales asociados al volcanismo y es usada culturalmente alterando sólo sus atributos formales), las arcillas, materia prima fundamental de la cerámica, son derivados secundarios de varios tipos de rocas y de sus combinaciones. Están ampliamente distribuidas en la naturaleza, están en constante proceso de alteración por causas naturales, y además son mezcladas por el hombre para generar un producto cultural alterando muchas veces la impronta original de la fuente de procedencia. Las arcillas a veces se limpian, otras veces se les agregan áridos, haciendo muy improbable reconocer la fuente original por comparación con depósitos naturales aún haciendo un catastro en una región. Agreguémosle a esto las alteraciones que sufren las vasijas con la manufactura, cocción, uso y eventuales procesos diagenéticos una vez que entran al depósito arqueológico.

Frente a las distintas metodologías que se han implementado en arqueología para superar los desafíos que impone la cerámica para reconocer su procedencia, nuestra opción ha sido utilizar una estrategia secuencial. A partir de una similitud formal constatada por análisis de formas y decoración, ésta parte con procedimientos clasificatorios de pasta generales, mediante observaciones a escala macroscópica (lupa binocular), que se pueden aplicar a una muestra importante del material de estudio y que cautelan una adecuada representatividad del conjunto cerámico. Sigue con la aplicación de técnicas analíticas más específicas que apuntan a la caracterización de esa muestra analizada con petrografía. Los costos y la especialización requerida en estos análisis hacen que se procesen cantidades reducidas de cerámica. Su objetivo es especificar los componentes minerales incluidos en las muestras y compararlas. Continuamos con procedimientos que buscan relacionar las pastas con el sustrato geológico regional y reconocer conjuntos que por sus perfiles químicos puedan inter pretarse como procedentes de una fuente común con INAA. Cada observación releva aspectos diferentes de la materialidad y es frecuente que, donde unos coinciden, otros divergen.

La conclusión más clara que sacamos de la experiencia trabajando con la cerámica en estas regiones es que, más que las virtudes particulares que pueda tener cada técnica, importa la acumulación sistemática de datos (programas de estudio) que permitan generar una base de información regional comparativa. Es esa acumulación la que, mediante recurrencia y reiteración, logra superar la heterogeneidad inherente a la alfarería. En nuestro caso, lo hemos conseguido a nivel de clasificación de patrones de pasta para el Período Alfarero Temprano de Chile central. Sin esa base, no habríamos logrado generar una propuesta con los materiales de El Indígeno. En petrografía tenemos un conjunto de datos aún insuficientes. Es necesario aumentar las muestras y aprender a reconocer aquellos detalles que, en nuestros sedimentos, resaltan las diferencias o aluden a una formación geológica particular. En el caso de la activación neutrónica, que es lo que hemos inte- 
grado más recientemente, tiene la ventaja de ser un procedimiento analítico estandarizado, relativamente rápido, con el que se puede determinar la concentración de diversos elementos químicos simultáneamente y procesar rápidamente desde un punto de vista estadístico, pero, al igual que en petrografía, la muestra es aún insuficiente.

De esta forma creemos que nuestra estrategia es una opción metodológica que está entregando buenos resultados y, al mismo tiempo, reconocemos que debemos seguir trabajando para sustentar nuestras hipótesis con argumentos más sólidos.
Agradecimientos Los trabajos se realizaron en el marco del Proyecto Fundación Andes-Antorchas $\mathrm{N}^{\circ}$ 13816-4 y contaron con la colaboración de Daniela Baudet en el análisis cerámico del sitio El Indígeno. Agradecemos a los Dres. Humberto Lagiglia, Adolfo Gil y Gustavo Neme por la disposición para que realizáramos estos estudios con material arqueológico de los sitios que ellos han investigado en Argentina, y por compartir gentilmente todos sus conocimientos. Del mismo modo agradecemos al Museo de Historia Natural de San Rafael y a su personal, por acogernos y brindarnos toda la ayuda necesaria.

\section{REFERENCIAS CITADAS}

ARNOLD, D. E., 1985. Ceramic theory and cultural process. Cambridge University Press, Cambridge.

FALABELLA, F., 2000. El sitio arqueológico de El Mercurio en el contexto de la problemática cultural del Período Alfarero Temprano en Chile central. Actas Segundo Taller de Arqueología de Chile central (1993), Http:// Members.Tripod.Cl/Lcbmchap /Ferfall.Htm.

FALABELLA, F. y O. ANDONIE, 2003. Regional ceramic production and distribution systems during the Late Intermediate Ceramic Period in central Chile based on neutron activation analyses. En Nuclear analytical techniques in archaeological investigations, M. Rossbach (Ed.), pp. 99-118. Technical Reports Series No 416, IAEA, Viena.

FALABELLA, F. y M. T. PLANELLA. 1979. Curso inferior del río Maipo: Evidencias agroalfareras. Tesis para obtener el Título de Arqueólogo, Departamento de Antropología, Facultad de Ciencias Sociales, Universidad de Chile, Santiago.

1987. Informe de investigaciones arqueológicas. Sitio Laguna El Peral-C. Actas del Primer Congreso de Antropología, pp. 526-539. Santiago.

FALABElLA, F., M. T. PLANELLA y B. TAGLE, 2001. Pipe e tradizione di fumare nelle società preispaniche del
Periodo Agroceramicolo Precoce nella regione centrale del Cile. Eleusis Nuova Serie 5: 137-52.

LAGIGLIA, H., 1997. Arqueología de cazadores recolectores cordilleranos de altura. Ediciones Ciencia y Arte, San Rafael, Mendoza.

PLANELLA, M. T., F. FALABELLA y B. TAGLE, 1996 Ms. Resultados de investig ación arqueológica de La Granja, años 1994 y 1995. Informe Proyecto FONDECYT 194-0457.

SANHUEZA, L., 1997. Relaciones 1lano-cordillera durante el Período Agroalfarero Temprano en Chile central: Una visión desde la cerámica. Tesis para obtener el Título de Arqueólogo, Departamento de Antropología, Facultad de Ciencias Sociales, Universidad de Chile, Santiago.

SANHUEZA, L., M. VASQUEZ y F. FALABELLA. 2003. Las sociedades alfareras tempranas de la cuenca de Santiago. Chungara 35 (1): 23-50.

VIVALLO, W., M. GARDEWEG, A. TASSARA, J. ZANETTINI, M. MARQUEZ y R. GONZALEZ, 1999. Mapa de recursos minerales del área fronteriza Argentino-Chilena entre los 34 y $56^{\circ} \mathrm{S}$. Publicación Geológica Multinacional $\mathrm{N}^{\circ} 1$, escala 1:1.000.000. SERNAGEOMIN y Servicio Geológico Minero Argentino. 